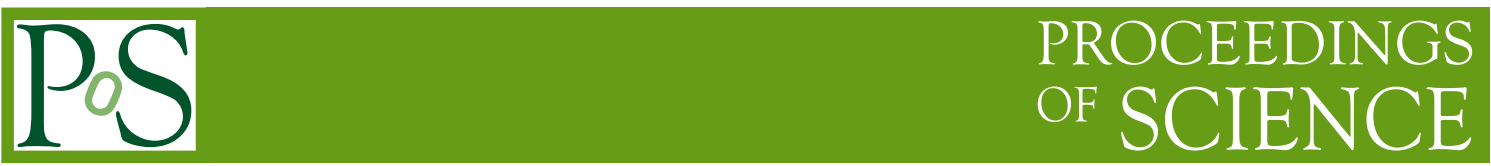

\title{
The ESSnuSB project
}

\section{Budimir Kliček ${ }^{\dagger, *}$}

Center of Excellence for Advanced Materials and Sensing Devices, Ruđer Bošković Institute, Bijenička cesta 54, 10000 Zagreb, Croatia

E-mail: budimir.klicek@irb.hr

Considering the relatively large value of the last measured neutrino mixing angle $\theta_{13}$, the way is now open to observe for the first time a possible $\mathrm{CP}$ violation in the leptonic sector. The measured value of $\theta_{13}$ also privileges the $2^{\text {nd }}$ oscillation maximum for the discovery of CP violation instead of the usually used $1^{\text {st }}$ oscillation maximum. The sensitivity to the $\mathrm{CP}$ violation signal at this $2^{\text {nd }}$ oscillation maximum is significantly higher than at the $1^{\text {st }}$ oscillation maximum thereby making the measurement significantly less sensitive to the systematic errors. Going to the $2^{\text {nd }}$ oscillation maximum necessitates a very intense neutrino beam with the appropriate energy. The world's most intense pulsed spallation neutron source, the European Spallation Source, will have a proton linac with the unprecedented power of $5 \mathrm{MW}$ and $2 \mathrm{GeV}$ energy. This linac, currently under construction, also has the potential to become the proton driver of the world's most intense neutrino beam with high potential to discover and well measure a neutrino CP violation. The physics performance of that neutrino Super Beam in conjunction with a megaton underground Water Cherenkov neutrino detector installed at a distance of about $500 \mathrm{~km}$ from ESS has been evaluated. In addition, the choice of such detector will extend the physics program to proton-decay, atmospheric neutrinos and astrophysics searches. The ESS proton linac upgrades, the accumulator ring needed for proton pulse compression, the target station optimization, and the physics potential are described. In addition to neutrinos, this facility will also produce at the same time a copious number of muons which could be used by a low energy nuSTORM facility, a Neutrino Factory or/and a muon collider. The ESS neutron facility will be ready by 2025 at which moment the upgrades for the neutrino facility could start.

40th International Conference on High Energy physics - ICHEP2020

July 28 - August 6, 2020

Prague, Czech Republic (virtual meeting)

\footnotetext{
*Speaker

${ }^{\dagger}$ On behalf of the ESSnuSB project.
} 


\section{Introduction}

The ESSnuSB is a design study for an experiment to measure the $\mathrm{CP}$ violation in the leptonic sector by observing neutrino oscillations. In particular, ESSnuSB aims to observe the difference in neutrino oscillation probabilities

$$
P_{v_{\mu} \rightarrow v_{e}} \neq P_{\bar{v}_{\mu} \rightarrow \bar{v}_{e}},
$$

where $P_{v_{\mu} \rightarrow v_{e}}$ and $P_{\bar{v}_{\mu} \rightarrow \bar{v}_{e}}$ are probabilities of oscillation of muon neutrinos to electron neutrinos and muon anti-neutrinos to electron anti-neutrinos, respectively.

It aims to do so by observing neutrino oscillations primarily at the $2^{\text {nd }}$ neutrino oscillation maximum, because the $\mathrm{CP}$ violation effect there is almost three times larger w.r.t. the $1^{\text {st }}$ maximum. It can be shown $[1,2]$ that

$$
\frac{\left(P_{v_{\mu} \rightarrow v_{e}}-P_{\bar{v}_{\mu} \rightarrow \bar{v}_{e}}\right) @ 2^{\text {nd }} \text { osc. max. }}{\left(P_{v_{\mu} \rightarrow v_{e}}-P_{\bar{v}_{\mu} \rightarrow \bar{v}_{e}}\right) @ 1^{\text {st } \text { osc. } \max .}} \sim 2.7,
$$

and that this ratio in vacuum depends only on neutrino mass splittings. This will make the experiment less sensitive to systematic errors, given that large enough statistical sample of neutrino interactions is retained when moving further away to reach the $2^{\text {nd }}$ maximum [3]. A high intensity neutrino beam is therefore required to fully utilize the advantages of such measurement.

To achieve a high intensity neutrino beam, ESSnuSB proposes to use the powerful ESS linac [4] as a proton driver. Once built, the linac will be the most powerful proton accelerator in the world, accelerating protons to $2.0 \mathrm{GeV}$ kinetic energy with an average beam power of $5 \mathrm{MW}$. Since ESS is designed to produce high intensity spallation neutron flux, a number of upgrades will be necessary to concurrently produce a neutrino beam.

A suite of near detectors will be placed close to the neutrino source with a purpose of measuring neutrino flux, its prompt flavour composition and neutrino-water interaction cross-sections. Two large water Cherenkov detectors, with a total fiducial mass of $540 \mathrm{kt}$ will be placed at a distance covering a large part of second neutrino oscillation maximum.

Such setup will have a significant discovery potential for the CP violation in the leptonic sector, and will allow for a precise measurement of the $\mathrm{CP}$ violation phase $\delta_{\mathrm{CP}}$.

\section{Linac upgrades and the target station}

In order to produce the neutrino beam concurrently with the spallation neutrons, the ESS linac will need to be modified.

The rate of the linac will need to be doubled from $14 \mathrm{~Hz}$ to $28 \mathrm{~Hz}$, and the proton kinetic energy will have to be increased from $2.0 \mathrm{GeV}$ to $2.5 \mathrm{GeV}$; this increase in energy will also benefit the neutron program. The ESSnuSB pulses will use $\mathrm{H}^{-}$ions instead of protons to facilitate the injection into the accumulator ring.

The accumulator ring with a circumference of about $400 \mathrm{~m}$ will be built to compress the long $2.86 \mathrm{~ms}$ ESS proton pulses to four short $1.3 \mu \mathrm{s}$ pulses. The short pulsed neutrino beam is necessary to sufficiently reduce the atmospheric neutrino background to enable the $\mathrm{CP}$ violation measurement. In addition, long ESS pulses would make the cooling of targets and magnetic horns very challenging. 
The target station will be composed of four neutrino production targets enveloped by magnetic horns. The multi-target design is necessary due to the cooling requirements set by the $5 \mathrm{MW}$ proton beam. In order to alternate the beam pulse between the targets, a dedicated beam switchyard will be built between the accumulator ring and the targets. A pion decay tunnel $25 \mathrm{~m}$ long and $4.0 \mathrm{~m}$ in diameter will be built downstream of the target station, ending with a hadron stop and muon monitors.

\section{Neutrino detectors}

The main purpose of near detectors is to measure the neutrino flux, its prompt flavour composition and neutrino-water interaction cross-sections. The measurement of cross-sections is of particular importance, since there is currently very little data on it in the ESSnuSB sub-GeV neutrino energy region (see Fig. 51.1 in [5]). To accomplish this, a suite of three detectors is proposed (in downstream to upstream order): a $0.5 \mathrm{kt}$ water Cherenkov detector, a $(1-4) \mathrm{t}$ SFGD scintillator detector similar to [6], and an emulsion detector with a water target similar to [7].

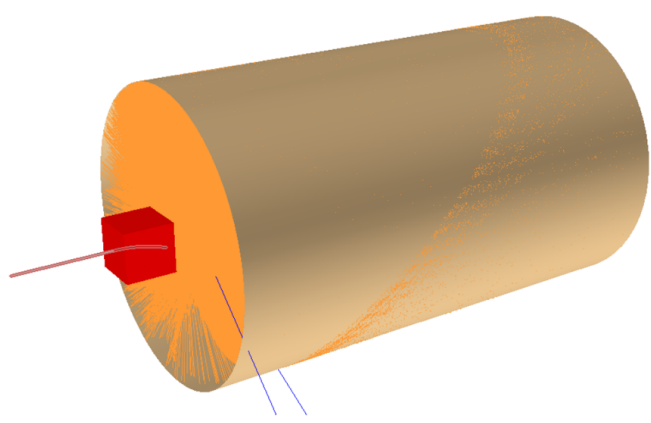

(a) Simulation of the near detector hall. A muon (orange track) is passing both through the SFGD detector (red cube) and the water Cherenkov detector immediately downstream.

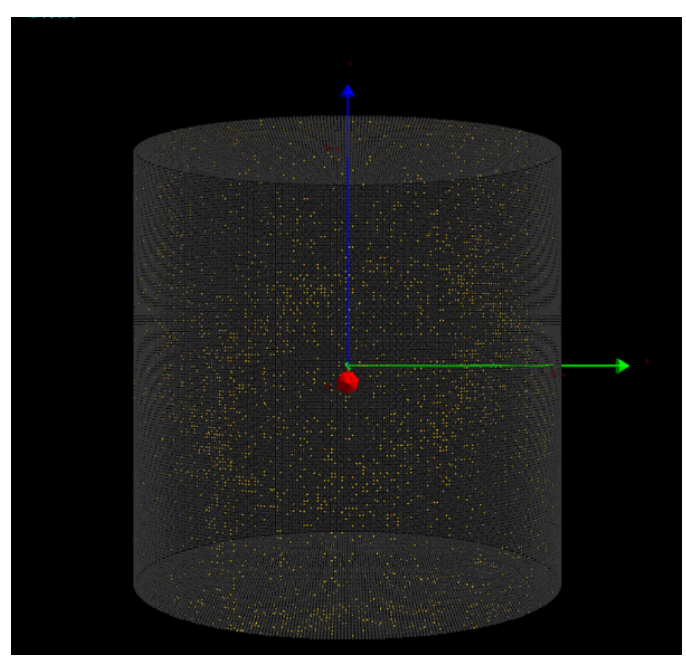

(b) Simulated neutrino interaction in one module of the far detector.

Figure 1: Simulation of near and far detectors in the ESSnuSB project.

There are two candidate sites for the far detectors: Zinkgruvan mine $360 \mathrm{~km}$ and Garpenberg mine $540 \mathrm{~km}$ away from the neutrino source. The Garpenberg mine covers the entire $2^{\text {nd }}$ oscillation maximum, while the Zinkgruvan mine covers partially both the $1^{\text {st }}$ and the $2^{\text {nd }}$ maximum and benefits from the increased expected number of neutrino interactions. Regardless of the site chosen, the far detector will be composed of two identical large water Cherenkov modules, each being a standing cylinder with a diameter of $D=78 \mathrm{~m}$ and height of $h=78 \mathrm{~m}$. The total water mass would therefore be $746 \mathrm{kt}$ with the fiducial target mass of $540 \mathrm{kt}$. The baseline design foresees using 20 inch photomultiplier tubes (PMTs) and an optical coverage of $30 \%$, which requires 72000 PMTs in total (both tanks). The price of PMTs located on the inner surface of the cylinder are the major driver of the total detector price. The $D=h$ option minimizes the surface to volume ratio of a cylinder and hence the number of PMTs required. The choice of having two detectors instead of 
one is mandated by immense technical difficulties to excavate a single cavern large enough to hold the entire desired volume of water.

\section{Physics reach}

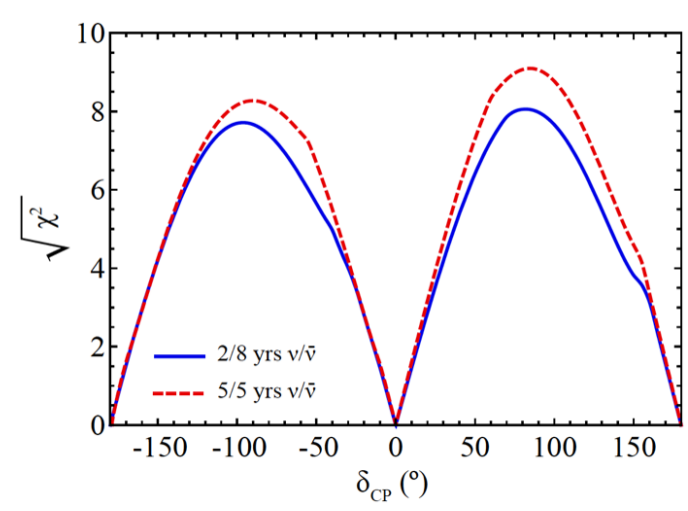

(a) Expected sensitivity of the ESSnuSB project

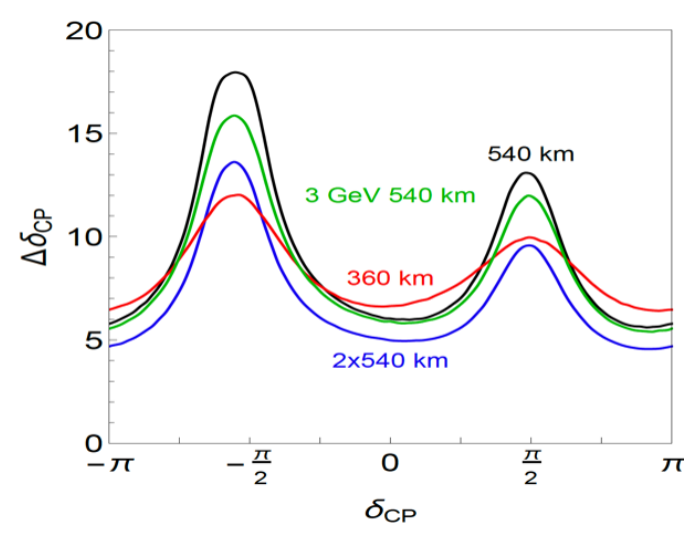

(b) Expected resolution of the ESSnuSB project

Figure 2: Expected sensitivity and resolution of the ESSnuSB project as a function of the CP violating angle $\delta_{\mathrm{CP}}$ after 10 years of data taking, before optimizations.

The expected physics reach of the ESSnuSB project is shown in Fig. 2. Since the measurement of the $\mathrm{CP}$ violating angle $\delta_{\mathrm{CP}}$ will be driven by neutrino oscillations at the $2^{\text {nd }}$ oscillation maximum, there is little dependence of projected sensitivity on the neutrino mass hierarchy. After 10 years of data taking, the experiment is expected to cover up to $60 \%$ of the $\delta_{\mathrm{CP}}$ range with sensitivity of more than $5 \sigma$. The resolution of $\delta_{\mathrm{CP}}$ measurement is expected to be down to $6^{\circ}$ at $\mathrm{CP}$ conserving angles $\delta_{\mathrm{CP}}=0^{\circ}$ and $\delta_{\mathrm{CP}}=180^{\circ}$.

\section{Synergies with future projects}

The upgrade of the ESS linac, especially the addition of the accumulator ring to produce $\mu$ s proton pulses, would lay a fertile ground for future developments of the high intensity frontier of particle physics. Some of the possibilities are schematically shown in Fig. 3.

Short pulses would allow observing the coherent neutrino scattering of neutrinos produced by pion decays at rest. The ESS linac in neutron mode will produce copious amount of such neutrinos, but the large duty cycle of $4 \%$ using long pulses would make it very difficult to discriminate between the external background and neutrino interactions. Short pulses would allow a significantly larger background rejection based on pulse timing.

Pions produced in the ESS neutrino mode could be injected in a nuSTORM-like race-track decay ring, where muons produced from their decays in the ring would continue circulating. Decays of these muons in the straight sections of the ring would produce a wide band beam of electron and muon neutrinos suitable for sterile neutrino search and electron neutrino interaction cross-section measurement (see [8]). 


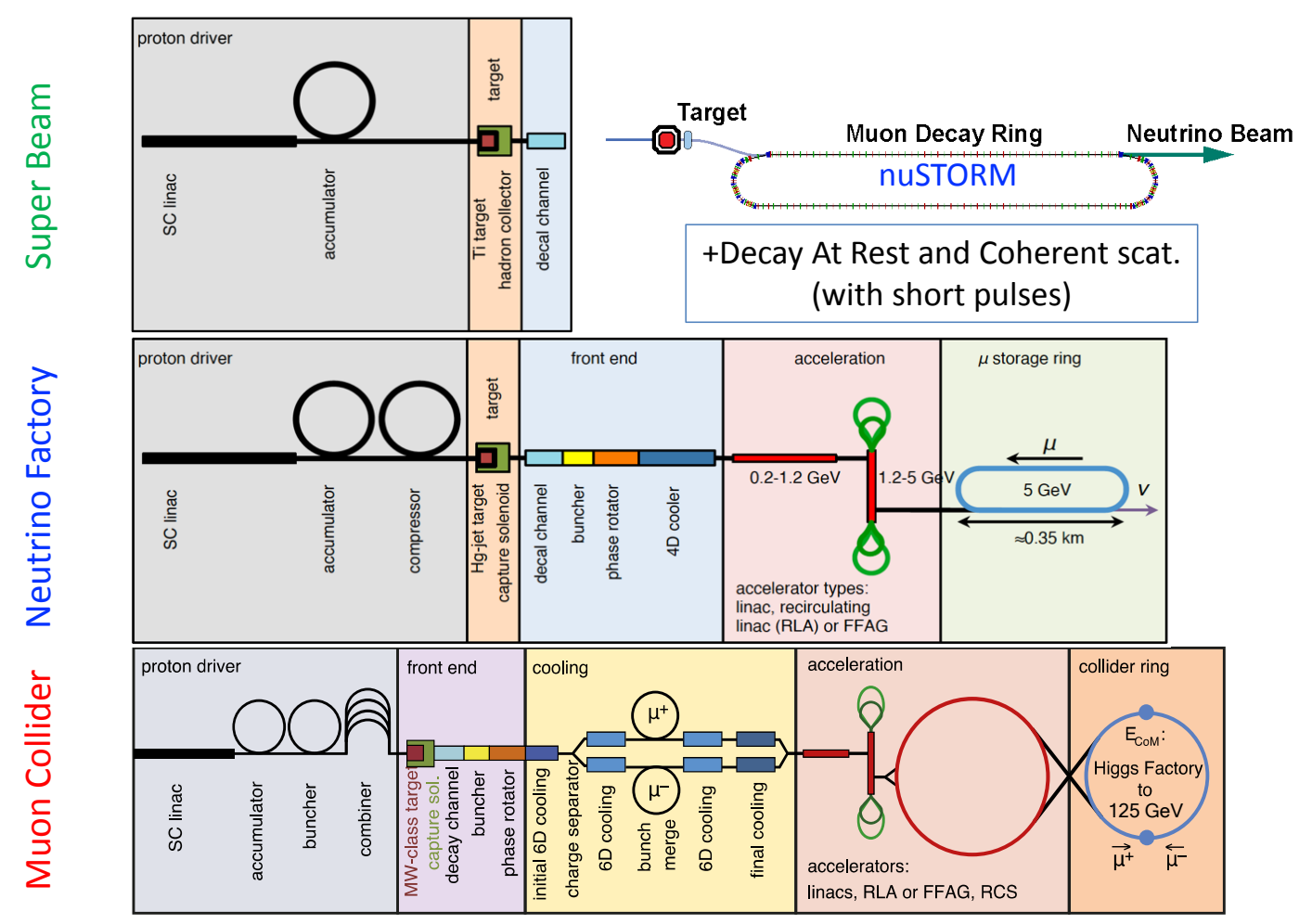

Figure 3: Possible synergies with future projects

Muons produced in the ESSnuSB decay tunnel or nuSTORM ring could be charge selected, cooled down and further accelerated. These accelerated muons could be then stored in a muon storage ring with straight sections. Muons decaying in straight sections would provide a narrow band $v_{\mu}$ and $v_{e}$ beam, as proposed by Neutrino Factory project [9].

As the most advanced option, muons of opposite charges could be collected, cooled and accelerated separately. These two muon beams will then be injected into a circular collider, where they will be accelerated further to $62.5 \mathrm{GeV}$, creating a first high energy muon collider. Used as a high intensitiy Higgs boson factory, the collider will allow a very precise measurement of its properties [10]. Unlike the proposed very large electron-positron colliders with similar scientific programs, the radius of this muon collider would be of the order of few hundred meters.

\section{Conclusions}

ESSnuSB aims to investigate CP violation in the lepton sector by observing $v_{\mu} \rightarrow v_{e}$ and $\bar{v}_{\mu} \rightarrow \bar{v}_{e}$ oscillations in the $2^{\text {nd }}$ oscillation maximum. This is made possible by using the uniquely powerful ESS linear accelerator as the proton driver in conjunction with the fact that $\theta_{13}$ is sufficiently large. Measurements at the second oscillation maximum benefit from the larger $\mathrm{CP}$ violation signal which makes them less susceptible to systematic errors. As an added bonus, oscillation probability at the $2^{\text {nd }}$ maximum is less dependent on the matter effects which could mimic the underlying $\mathrm{CP}$ 
violation. This makes ESSnuSB an ideal experiment in the future precision era of leptonic $\mathrm{CP}$ violation measurement.

The proposed modifications to the ESS linac, especially the introduction of an accumulator ring to compress proton pulses, open new possibilities for additional physics programs. This include coherent neutrino scattering experiments, nuSTORM-like muon decay rings, Neutrino Factory, muon collider and more.

\section{Acknowledgments}

This project is supported by the COST Action CA15139 "Combining forces for a novel European facility for neutrino-antineutrino symmetry-violation discovery" (EuroNuNet). It has also received funding from the European Union's Horizon 2020 research and innovation programme under grant agreement No 777419.

\section{References}

[1] H. Nunokawa, S.J. Parke and J.W. Valle, CP Violation and Neutrino Oscillations, Prog. Part. Nucl. Phys. 60 (2008) 338 [0710.0554].

[2] S. Parke, Neutrinos: Theory and Phenomenology, Phys. Scripta T 158 (2013) 014013 [1310.5992].

[3] P. Coloma and E. Fernandez-Martinez, Optimization of neutrino oscillation facilities for large $\theta_{13}$, JHEP 04 (2012) 089 [1110 . 4583].

[4] R. Garoby, A. Vergara, H. Danared, I. Alonso, E. Bargallo, B. Cheymol et al., The European Spallation Source Design, Physica Scripta 93 (2017) 014001.

[5] P. Zyla et al., Review of Particle Physics, PTEP 2020 (2020) 083C01.

[6] A. Blondel et al., A fully active fine grained detector with three readout views, JINST 13 (2018) P02006 [1707.01785].

[7] A. Hiramoto et al., First measurement of $\bar{v}_{\mu}$ and $v_{\mu}$ charged-current inclusive interactions on water using a nuclear emulsion detector, Phys. Rev. D 102 (2020) 072006 [2008. 03895].

[8] D. Adey et al., nuSTORM - Neutrinos from STORed Muons: Proposal to the Fermilab PAC, 1308.6822.

[9] M. Bogomilov et al., Neutrino Factory, Phys. Rev. ST Accel. Beams 17 (2014) 121002.

[10] C. Rubbia, Further searches of the Higgs scalar sector at the ESS, 1908.05664v3. 